\title{
THE BEURLING OPERATOR FOR THE HYPERBOLIC PLANE
}

\author{
Håkan Hedenmalm \\ The Royal Institute of Technology, Department of Mathematics \\ S-100 44 Stockholm, Sweden; haakanh@math.kth.se
}

\begin{abstract}
We find a Beurling operator for the hyperbolic plane, and obtain an $L^{2}$ norm identity for it, as well as two-sided $L^{p}$ estimates.
\end{abstract}

\section{Introduction and statement of main results}

1.1. Outline of the paper. We first mention the classical Cauchy and Beurling operators $\mathbf{C}$ and $\mathbf{B}$ in the setting of the plane. We then introduce their hyperbolic plane analogues $\mathbf{C}^{\uparrow}, \mathbf{C}^{\downarrow}$ and $\mathbf{B}^{\uparrow}, \mathbf{B}^{\downarrow}$. For instance, the hyperbolic Cauchy operator $\mathbf{C}^{\downarrow}$ finds the $L^{2}$-minimal solution to the $\bar{\partial}$-problem. The mapping properties of $\mathbf{C}^{\uparrow}, \mathbf{C}^{\downarrow}$ rely on the well-known Hardy inequality for the upper half plane. We find a sharp two-sided estimate for the the norm of $\mathbf{B}^{\downarrow}[f]$ in weighted $L^{p}$-spaces, which is analogous to the well-known two-sided estimate for $\mathbf{B}[f]$. In the Hilbert space case $p=2$, the estimate becomes a norm isometry. The way the proof is set up, we need some results of Liouville type for the hyperbolic plane. In the final section, we try to explain the assertion of the main theorem in geometric terms.

1.2. The Beurling transform. The (Ahlfors-)Beurling transform (or operator) $\mathbf{B}: L^{2}(\mathbb{C}) \rightarrow L^{2}(\mathbb{C})$ is formally the operator $\mathbf{B}=\boldsymbol{\partial} \bar{\partial}^{-1}$. Here, we use the notation

$$
\boldsymbol{\partial}_{z}:=\frac{1}{2}\left(\frac{\partial}{\partial x}-\mathrm{i} \frac{\partial}{\partial y}\right), \quad \overline{\boldsymbol{\partial}}_{z}:=\frac{1}{2}\left(\frac{\partial}{\partial x}+\mathrm{i} \frac{\partial}{\partial y}\right),
$$

and put $\boldsymbol{\Delta}_{z}:=\boldsymbol{\partial}_{z} \overline{\boldsymbol{\partial}}_{z} ;$ as above, we frequently suppress the subscript $z$. This way of defining $\mathbf{B}$ leaves some ambiguity, as there are many possible ways to define $\overline{\boldsymbol{\partial}}^{-1}$. The standard choice is to use the Cauchy transform $\mathbf{C}$ for $\overline{\boldsymbol{\partial}}^{-1}$,

$$
\mathbf{C}[f](z):=\int_{\mathbb{C}} \frac{f(w)}{z-w} \mathrm{~d} A(w), \quad z \in \mathbb{C},
$$

where

$$
\mathrm{d} A(z):=\frac{\mathrm{d} x \mathrm{~d} y}{\pi}, \quad z=x+\mathrm{i} y
$$

is normalized area measure. Unfortunately, the integral defining $\mathbf{C}[f]$ is not welldefined for all $f \in L^{2}(\mathbb{C})$, but at least when $f$ is compactly supported, there is no

doi:10.5186/aasfm.2012.3708

2010 Mathematics Subject Classification: Primary 30E20, 42B20.

Key words: Beurling operator, isometry, norm estimates, hyperbolic plane.

Research partially supported by the Göran Gustafsson (KVA) Foundation and by the Swedish Science Council (Vetenskapsrådet). 
problem. Differentiating the Cauchy transform, we get

$$
\mathbf{B}[f](z):=-\mathrm{pv} \int_{\mathbb{C}} \frac{f(w)}{(z-w)^{2}} \mathrm{~d} A(w), \quad z \in \mathbb{C},
$$

where "pv" stands for the principal value. It is easy to show, using Fourier analysis or Green's formula, that B acts isometrically on $L^{2}(\mathbb{C})$ :

$$
\|\mathbf{B}[f]\|_{L^{2}(\mathbb{C})}^{2}=\|f\|_{L^{2}(\mathbb{C})}^{2}=\int_{\mathbb{C}}|f|^{2} \mathrm{~d} A,
$$

where the rightmost identity defines the norm in $L^{2}(\mathbb{C})$. It is well-known that $\mathbf{B}$ acts boundedly on $L^{p}(\mathbb{C})$ for $1<p<+\infty$; let $B(p)$ denote its norm, that is, the best constant such that

$$
\|\mathbf{B}[f]\|_{L^{p}(\mathbb{C})} \leq B(p)\|f\|_{L^{p}(\mathbb{C})}, \quad f \in L^{p}(\mathbb{C}),
$$

holds. It is easy to show that there is an estimate from below as well:

$$
\frac{1}{B(p)}\|f\|_{L^{p}(\mathbb{C})} \leq\|\mathbf{B}[f]\|_{L^{p}(\mathbb{C})} \leq B(p)\|f\|_{L^{p}(\mathbb{C})}, \quad f \in L^{p}(\mathbb{C}) .
$$

A well-known conjecture due to Iwaniec (see [9], [3], [7], [4]) claims that

$$
B(p)=\max \left\{p-1, \frac{1}{p-1}\right\}, \quad 1<p<+\infty .
$$

An easy duality argument shows that with $p^{\prime}=p /(p-1)$ (dual exponent),

$$
B(p)=B\left(p^{\prime}\right), \quad 1<p<+\infty .
$$

We mention that there is a formulation of (1.2) which does not use singular integrals:

$$
\frac{1}{B(p)}\|\overline{\boldsymbol{\partial}} g\|_{L^{p}(\mathbb{C})} \leq\|\boldsymbol{\partial} g\|_{L^{p}(\mathbb{C})} \leq B(p)\|\overline{\boldsymbol{\partial}} g\|_{L^{p}(\mathbb{C})}, \quad g \in C_{c}^{\infty}(\mathbb{C}),
$$

where $C_{c}^{\infty}(\mathbb{C})$ is the space of compactly supported test functions.

1.3. The hyperbolic plane. Let $\mathbb{H}$ denote the hyperbolic plane; we shall use the model

where

$$
\mathbb{H}=\left\langle\mathbb{C}_{+}, \mathrm{d} s_{\mathbb{H}}\right\rangle
$$

is the upper half plane, and

$$
\mathbb{C}_{+}=\{z \in \mathbb{C}: \operatorname{Im} z>0\}
$$

$$
\mathrm{d} s_{\mathbb{H}}(z)=\frac{|\mathrm{d} z|}{\operatorname{Im} z}
$$

is the Poincaré metric. The corresponding hyperbolic area element is given by

$$
\mathrm{d} A_{\mathbb{H}}(z)=\frac{\mathrm{d} A(z)}{(\operatorname{Im} z)^{2}} .
$$

1.4. The function spaces. For $0<p<+\infty$ and real $q$, we introduce the space $L_{q}^{p}\left(\mathbb{C}_{+}\right)$of (equivalence classes of) area-Lebesgue measurable functions subject to the integrability condition

$$
\|f\|_{L_{q}^{p}\left(\mathbb{C}_{+}\right)}^{p}=\int_{\mathbb{C}_{+}}|f(z)|^{p}(\operatorname{Im} z)^{q} \mathrm{~d} A(z)=\int_{\mathbb{C}_{+}}|f(z)|^{p}(\operatorname{Im} z)^{q+2} \mathrm{~d} A_{\mathbb{H}}(z)<+\infty .
$$

It is a Banach space for $1 \leq p<+\infty$. We realize that $L_{-2}^{2}\left(\mathbb{C}_{+}\right)$has the interpretation of $L^{2}(\mathbb{H})$, the $L^{2}$ space over the hyperbolic plane. 
1.5. Some notation. We shall at times need conjugate symbol operators, as defined by

$$
\overline{\mathbf{T}}[f]=\operatorname{conj}(\mathbf{T}[\bar{f}])
$$

and we apply this notational convention to all the operators considered here. Moreover, if $\mathcal{F}$ is a collection of complex-valued functions, we write $\operatorname{conj}(\mathcal{F})$ for the collection of complex conjugates of the functions in $\mathcal{F}$.

1.6. Hardy's inequality for the upper half plane. By Hardy's inequality for the upper half space,

$$
\int_{\mathbb{C}_{+}}|f(z)|^{p} \frac{\mathrm{d} A(z)}{(\operatorname{Im} z)^{p}} \leq 2^{p / 2}(1-1 / p)^{-p} \int_{\mathbb{C}_{+}}\left(|\boldsymbol{\partial} f(z)|^{2}+|\overline{\boldsymbol{\partial}} f(z)|^{2}\right)^{p / 2} \mathrm{~d} A(z)
$$

for $f \in C_{c}^{\infty}\left(\mathbb{C}_{+}\right)$. The constant is sharp (see, e.g., [6], [11]). If we use that for $a, b \in \mathbb{C}$,

$$
\left(|a|^{2}+|b|^{2}\right)^{p / 2} \leq A(p)\left(|a|^{p}+|b|^{p}\right), \quad A(p):=\max \left\{1,2^{-1+p / 2}\right\},
$$

we get

$$
\int_{\mathbb{C}_{+}}|f(z)|^{p} \frac{\mathrm{d} A(z)}{(\operatorname{Im} z)^{p}} \leq 2^{p / 2}(1-1 / p)^{-p} A(p) \int_{\mathbb{C}_{+}}\left(|\boldsymbol{\partial} f(z)|^{p}+|\overline{\boldsymbol{\partial}} f(z)|^{p}\right) \mathrm{d} A(z),
$$

which in terms of norms reads

$$
\|f\|_{L_{-p}^{p}\left(\mathbb{C}_{+}\right)}^{p} \leq 2^{p / 2}(1-1 / p)^{-p} A(p)\left(\|\boldsymbol{\partial} f\|_{L^{p}\left(\mathbb{C}_{+}\right)}^{p}+\|\overline{\boldsymbol{\partial}} f\|_{L^{p}\left(\mathbb{C}_{+}\right)}^{p}\right), \quad f \in C_{c}^{\infty}\left(\mathbb{C}_{+}\right) .
$$

Next, since by (1.2),

$$
\|\boldsymbol{\partial} f\|_{L^{p}\left(\mathbb{C}_{+}\right)} \leq B(p)\|\bar{\partial} f\|_{L^{p}\left(\mathbb{C}_{+}\right)}, \quad f \in C_{c}^{\infty}\left(\mathbb{C}_{+}\right),
$$

we obtain from (1.5) that

$$
\|f\|_{L_{-p}^{p}\left(\mathbb{C}_{+}\right)}^{p} \leq 2^{p / 2}(1-1 / p)^{-p} A(p)\left(1+B(p)^{p}\right)\|\overline{\boldsymbol{\partial}} f\|_{L^{p}\left(\mathbb{C}_{+}\right)}^{p}, \quad f \in C_{c}^{\infty}\left(\mathbb{C}_{+}\right) .
$$

It is not obvious whether the constant appearing on the right hand side of (1.6) is optimal for general $p$. However, in case $p=2,(1.6)$ reads

$$
\|f\|_{L_{-2}^{2}\left(\mathbb{C}_{+}\right)} \leq 4\|\bar{\partial} f\|_{L^{2}\left(\mathbb{C}_{+}\right)}, \quad f \in C_{c}^{\infty}\left(\mathbb{C}_{+}\right),
$$

and the constant is sharp.

1.7. The Cauchy operators associated with the upper half plane. For functions $f$ defined on $\mathbb{C}_{+}$, we introduce the Cauchy-type operators

$$
\mathbf{C}^{\downarrow}[f](z):=\int_{\mathbb{C}_{+}}\left[\frac{1}{z-w}-\frac{1}{z-\bar{w}}\right] f(w) \mathrm{d} A(w)=2 \mathrm{i} \int_{\mathbb{C}_{+}} \frac{f(w) \operatorname{Im} w}{(z-w)(z-\bar{w})} \mathrm{d} A(w),
$$

$z \in \mathbb{C}_{+}$, and

$$
\mathbf{C}^{\uparrow}[f](z):=\int_{\mathbb{C}_{+}}\left[\frac{1}{z-w}-\frac{1}{\bar{z}-w}\right] f(w) \mathrm{d} A(w)=-2 \mathrm{i} \int_{\mathbb{C}_{+}} \frac{\operatorname{Im} z f(w)}{(z-w)(\bar{z}-w)} \mathrm{d} A(w),
$$

$z \in \mathbb{C}_{+}$, for all locally integrable functions $f$ for which the integrals make sense (almost everywhere on $\mathbb{C}_{+}$). The operator $\mathbf{C}^{\downarrow}$ appears in the context of the unit disk in Subsection 4.8.3 of the book [2] by Astala, Iwaniec, Martin. The identity

$$
\left(\frac{1}{z-w}-\frac{1}{z-\bar{w}}\right)+\left(\frac{1}{\bar{z}-\bar{w}}-\frac{1}{\bar{z}-w}\right)=\left(\frac{1}{z-w}-\frac{1}{\bar{z}-w}\right)+\left(\frac{1}{\bar{z}-\bar{w}}-\frac{1}{z-\bar{w}}\right)
$$


entails the operator identity

$$
\mathbf{C}^{\downarrow}+\overline{\mathbf{C}}^{\downarrow} \equiv \mathbf{C}^{\uparrow}+\overline{\mathbf{C}}^{\uparrow}
$$

Moreover, with respect to the inner product of $L^{2}\left(\mathbb{C}_{+}\right)$, we have that

$$
\left(\mathbf{C}^{\downarrow}\right)^{*}=-\overline{\mathbf{C}}^{\uparrow}, \quad\left(\overline{\mathbf{C}}^{\downarrow}\right)^{*}=-\mathbf{C}^{\uparrow}, \quad\left(\mathbf{C}^{\uparrow}\right)^{*}=-\overline{\mathbf{C}}^{\downarrow}, \quad\left(\overline{\mathbf{C}}^{\uparrow}\right)^{*}=-\mathbf{C}^{\downarrow} .
$$

To understand the action of $\mathbf{C}^{\uparrow}$, we note that

$$
\begin{aligned}
F(z)-\mathbf{C}^{\uparrow}[\overline{\boldsymbol{\partial}} F](z) & =F(z)-\int_{\mathbb{C}_{+}}\left(\frac{1}{z-w}-\frac{1}{\bar{z}-w}\right) \bar{\partial} F(w) \mathrm{d} A(w) \\
& =\int_{\mathbb{C}_{+}} \bar{\partial}_{w}\left\{\left(\frac{1}{w-z}-\frac{1}{w-\bar{z}}\right) F(w)\right\} \mathrm{d} A(w) \\
& =\frac{1}{2 \pi \mathrm{i}} \int_{\mathbb{R}}\left(\frac{1}{w-z}-\frac{1}{w-\bar{z}}\right) F(w) \mathrm{d} w, \quad z \in \mathbb{C}_{+},
\end{aligned}
$$

provided $F$ and $\overline{\boldsymbol{\partial}} F$ are smooth and taper off relatively quickly to 0 at infinity (the middle integral is to be interpreted in the sense of distribution theory). As a first application of (1.10), we find that

$$
\mathbf{C}^{\uparrow}[\overline{\boldsymbol{\partial}} f]=f, \quad f \in C_{0}^{\infty}\left(\mathbb{C}_{+}\right) .
$$

The $L^{2}\left(\mathbb{C}_{+}\right)$-closure of $\bar{\partial} C_{c}^{\infty}\left(\mathbb{C}_{+}\right)$equals $L^{2}\left(\mathbb{C}_{+}\right) \ominus \operatorname{conj}\left(A^{2}\left(\mathbb{C}_{+}\right)\right)$(this fact is known as Havin's lemma). A second application of (1.10) shows that

$$
\mathbf{C}^{\uparrow}[g]=0, \quad g \in \operatorname{conj}\left(A^{2}\left(\mathbb{C}_{+}\right)\right),
$$

which means that we have determined the action of $\mathbf{C}^{\uparrow}$ on all of $L^{2}\left(\mathbb{C}_{+}\right)$. It now follows from (1.11) and (1.12) combined with (1.7) that

$$
\left\|\mathbf{C}^{\uparrow}[g]\right\|_{L_{-2}^{2}\left(\mathbb{C}_{+}\right)} \leq 4\|g\|_{L^{2}\left(\mathbb{C}_{+}\right)}, \quad g \in L^{2}\left(\mathbb{C}_{+}\right) .
$$

Expressed differently, the operator

$$
\mathbf{C}^{\uparrow}: L^{2}\left(\mathbb{C}_{+}\right) \rightarrow L_{-2}^{2}\left(\mathbb{C}_{+}\right)
$$

is bounded and has norm 4. A similar argument based on (1.6) shows that

$$
\mathbf{C}^{\uparrow}: L^{p}\left(\mathbb{C}_{+}\right) \rightarrow L_{-p}^{p}\left(\mathbb{C}_{+}\right), \quad 1<p<+\infty,
$$

with a norm bound which depends on $p$. Let $C^{\uparrow}(p)$ be the norm of this operator. By (1.9), and the fact that with respect to the inner product of $L^{2}\left(\mathbb{C}_{+}\right)$, the spaces $L_{-p}^{p}\left(\mathbb{C}_{+}\right)$and $L_{p^{\prime}}^{p^{\prime}}\left(\mathbb{C}_{+}\right)$are dual to one another (here, $p^{\prime}=p /(p-1)$ ), we have that

$$
\mathbf{C}^{\downarrow}: L_{p}^{p}\left(\mathbb{C}_{+}\right) \rightarrow L^{p}\left(\mathbb{C}_{+}\right), \quad 1<p<+\infty,
$$

is bounded as well; we denote its norm by $C^{\downarrow}(p)$. The duality argument actually gives that

$$
C^{\downarrow}(p)=C^{\uparrow}\left(p^{\prime}\right), \quad p^{\prime}=p /(p-1) .
$$

As noted previously, for $p=2$, we have $C^{\downarrow}(2)=C^{\uparrow}(2)=4$.

We specialize for a moment to $p=2$ and look for an interpretation of the operator $\mathbf{C}^{\downarrow}$. By duality, the information on the null space of $\mathbf{C}^{\uparrow}$ supplied by (1.12) leads to information on the range of $\mathbf{C}^{\downarrow}$ :

$$
\mathbf{C}^{\downarrow}: L_{2}^{2}\left(\mathbb{C}_{+}\right) \rightarrow L^{2}\left(\mathbb{C}_{+}\right) \ominus A^{2}\left(\mathbb{C}_{+}\right) .
$$


The operator $\mathbf{C}^{\downarrow}$ therefore furnishes the least norm solution to the $\overline{\boldsymbol{\partial}}$-problem: $u=$ $\mathbf{C}^{\downarrow}[f]$ has smallest norm in $L^{2}\left(\mathbb{C}_{+}\right)$among all solutions to

$$
\bar{\partial} u=f(z), \quad z \in \mathbb{C}_{+} .
$$

1.8. Double singularity Cauchy-type integral operators. We introduce the operators $\mathbf{D}^{\uparrow}, \mathbf{D}^{\downarrow}$, as given by

$$
\mathbf{D}^{\uparrow}[g](z):=\int_{\mathbb{C}_{+}} \frac{g(w)}{(z-w)(\bar{z}-w)} \mathrm{d} A(w), \quad z \in \mathbb{C}_{+},
$$

and

$$
\mathbf{D}^{\downarrow}[g](z):=\int_{\mathbb{C}_{+}} \frac{g(w)}{(z-w)(z-\bar{w})} \mathrm{d} A(w), \quad z \in \mathbb{C}_{+} .
$$

We readily check that

$$
\mathbf{C}^{\uparrow}=-2 \mathrm{i} \mathbf{M} \mathbf{D}^{\uparrow}, \quad \mathbf{C}^{\downarrow}=2 \mathrm{i} \mathbf{D}^{\downarrow} \mathbf{M},
$$

where $\mathbf{M}[f](z)=(\operatorname{Im} z) f(z)$. The analogous operators in the setting of the unit disk $\mathbf{D}$ in place of $\mathbb{C}_{+}$appeared recently in [5]. The boundedness of the operators $\mathbf{C}^{\uparrow}$ and $\mathrm{C}^{\downarrow}$ in the corresponding contexts entails that

$$
\mathbf{D}^{\uparrow}: L^{p}\left(\mathbb{C}_{+}\right) \rightarrow L^{p}\left(\mathbb{C}_{+}\right), \quad \mathbf{D}^{\downarrow}: L^{p}\left(\mathbb{C}_{+}\right) \rightarrow L^{p}\left(\mathbb{C}_{+}\right),
$$

act boundedly for $1<p<+\infty$. Moreover, the norms of these operators may be expressed in terms of $C^{\uparrow}(p), C^{\downarrow}(p)$. In [5], the operators $\mathbf{D}^{\uparrow}$ and $\mathbf{D}^{\downarrow}$ appeared in the analysis of conformal maps. We may bring the analysis one step further and consider, for a conformal mapping $\varphi: \mathbb{C}_{+} \rightarrow \Omega$, where $\Omega \subset \mathbb{C}$, the operators

$$
\mathbf{D}_{\varphi}^{\uparrow}[g](z)=\int_{\mathbb{C}_{+}} \frac{\varphi^{\prime}(z) g(w)}{(\varphi(z)-\varphi(w))(\bar{z}-w)} \mathrm{d} A(w), \quad z \in \mathbb{C}_{+},
$$

and

$$
\mathbf{D}_{\varphi}^{\downarrow}[g](z)=\int_{\mathbb{C}_{+}} \frac{\varphi^{\prime}(w) g(w)}{(\varphi(z)-\varphi(w))(z-\bar{w})} \mathrm{d} A(w), \quad z \in \mathbb{C}_{+} .
$$

The instance $\varphi(z)=z$ gives us the operators $\mathbf{D}^{\uparrow}, \mathbf{D}^{\downarrow}$ already mentioned. The more general operators $\mathbf{D}_{\varphi}^{\uparrow}, \mathbf{D}_{\varphi}^{\downarrow}$ deserve attention as well (cf. [5]).

1.9. The sum of two Cauchy-type operators. The identity (1.8) suggests that we should study the operator

$$
\mathbf{C}_{\text {sum }}:=\mathbf{C}^{\downarrow}+\overline{\mathbf{C}}^{\downarrow}=\mathbf{C}^{\uparrow}+\overline{\mathbf{C}}^{\uparrow} .
$$

The mapping properties of $\mathbf{C}^{\downarrow}$ and $\mathbf{C}^{\uparrow}$ show that $\mathbf{C}_{\text {sum }}$ maps boundedly $(1<p<+\infty)$

$$
\mathbf{C}_{\text {sum }}: L_{p}^{p}\left(\mathbb{C}_{+}\right) \rightarrow L^{p}\left(\mathbb{C}_{+}\right), \quad \mathbf{C}_{\text {sum }}: L^{p}\left(\mathbb{C}_{+}\right) \rightarrow L_{-p}^{p}\left(\mathbb{C}_{+}\right) .
$$

Interpolation theory allows us to combine these statements to get that $\mathbf{C}_{\text {sum }}$ maps boundedly $(1<p<+\infty)$

$$
\mathbf{C}_{\text {sum }}: L_{q}^{p}\left(\mathbb{C}_{+}\right) \rightarrow L_{q-p}^{p}\left(\mathbb{C}_{+}\right), \quad 0 \leq q \leq p .
$$

The calculation

$$
\left(\frac{1}{z-w}-\frac{1}{z-\bar{w}}\right)+\left(\frac{1}{\bar{z}-\bar{w}}-\frac{1}{\bar{z}-w}\right)=8 \operatorname{Im} z \operatorname{Im} w \frac{\operatorname{Re}(z-w)}{|(z-w)(z-\bar{w})|^{2}}
$$

shows that

$$
\mathbf{C}_{\text {sum }}=8 \mathrm{MEM}
$$


where $\mathbf{E}$ is the integral operator

$$
\mathbf{E}[f](z):=\int_{\mathbb{C}_{+}} \frac{\operatorname{Re}(z-w)}{|(z-w)(z-\bar{w})|^{2}} f(w) \mathrm{d} A(w), \quad z \in \mathbb{C}_{+} .
$$

We read off from the mapping property of $\mathbf{C}_{\text {sum }}$ that $\mathbf{E}$ acts boundedly $(1<p<+\infty)$

$$
\mathbf{E}: L_{q-p}^{p}\left(\mathbb{C}_{+}\right) \rightarrow L_{q}^{p}\left(\mathbb{C}_{+}\right), \quad 0 \leq q \leq p .
$$

For $p=2$ we even have that $\mathbf{E}: L_{q-2}^{2}\left(\mathbb{C}_{+}\right) \rightarrow L_{q}^{2}\left(\mathbb{C}_{+}\right)$is norm contraction for $0 \leq$ $q \leq 2$.

\section{The Beurling transform for the hyperbolic plane}

2.1. The Beurling-type operators. We introduce the Beurling-type operators

$$
\mathbf{B}^{\downarrow}[f](z):=\boldsymbol{\partial} \mathbf{C}^{\downarrow}[f](z)=\operatorname{pv} \int_{\mathbb{C}_{+}}\left[\frac{1}{(z-\bar{w})^{2}}-\frac{1}{(z-w)^{2}}\right] f(w) \mathrm{d} A(w), \quad z \in \mathbb{C}_{+},
$$

and

$$
\mathbf{B}^{\uparrow}[f](z):=\operatorname{pv} \int_{\mathbb{C}_{+}}\left[\frac{1}{(\bar{z}-w)^{2}}-\frac{1}{(z-w)^{2}}\right] f(w) \mathrm{d} A(w), \quad z \in \mathbb{C}_{+},
$$

for functions $f$ such that the above expressions make sense. With respect to the inner product of $L^{2}\left(\mathbb{C}_{+}\right)$, we have the adjoint calculation formulas

$$
\left(\mathbf{B}^{\downarrow}\right)^{*}=\overline{\mathbf{B}}^{\uparrow}, \quad\left(\overline{\mathbf{B}}^{\downarrow}\right)^{*}=\mathbf{B}^{\uparrow}, \quad\left(\mathbf{B}^{\uparrow}\right)^{*}=\overline{\mathbf{B}}^{\downarrow}, \quad\left(\overline{\mathbf{B}}^{\downarrow}\right)^{*}=\mathbf{B}^{\uparrow} .
$$

In analogy with (1.8), we have the operator identity

$$
\mathbf{B}^{\downarrow}+\overline{\mathbf{B}}^{\downarrow}=\mathbf{B}^{\uparrow}+\overline{\mathbf{B}}^{\downarrow} \text {. }
$$

If we extend $f$ to $\mathbb{C}$ by declaring it to vanish off $\mathbb{C}_{+}$, we have

$$
\mathbf{B}^{\downarrow}[f](z)=\mathbf{B}[f](z)-\overline{\mathbf{B}}[f](\bar{z}), \quad z \in \mathbb{C}_{+},
$$

and

$$
\mathbf{B}^{\uparrow}[f](z)=\mathbf{B}[f](z)-\mathbf{B}[f](\bar{z}), \quad z \in \mathbb{C}_{+} .
$$

In view of (1.2), we see that $\mathbf{B}^{\downarrow}$ and $\mathbf{B}^{\uparrow}$ act boundedly on $L^{p}\left(\mathbb{C}_{+}\right)$for $1<p<+\infty$, with norm bound

$$
\left\|\mathbf{B}^{\downarrow}[f]\right\|_{L^{p}\left(\mathbb{C}_{+}\right)} \leq 2 B(p)\|f\|_{L^{p}\left(\mathbb{C}_{+}\right)}, \quad f \in L^{p}\left(\mathbb{C}_{+}\right) .
$$

The analogous bound holds for $\mathbf{B}^{\uparrow}$ as well.

We shall obtain a more interesting result. For $1<p<+\infty$, $\mathbf{B}^{\downarrow}$ acts boundedly on $L_{p}^{p}\left(\mathbb{C}_{+}\right)$, while $\mathbf{B}^{\uparrow}$ acts boundedly on $L_{-p}^{p}\left(\mathbb{C}_{+}\right)$. It should be pointed out here that the functions in $L_{p}^{p}\left(\mathbb{C}_{+}\right)$, extended to vanish on $\mathbb{C} \backslash \mathbb{C}_{+}$, need not be locally area-integrable near the real line, and therefore it is not clear how, e.g., the operator B could be defined on $L_{p}^{p}\left(\mathbb{C}_{+}\right)$. But $\mathbf{B}^{\downarrow}$ is well-defined due to the cancellation in the symbol.

The spaces $L_{p}^{p}\left(\mathbb{C}_{+}\right)$and $L_{-p^{\prime}}^{p^{\prime}}\left(\mathbb{C}_{+}\right)$are dual to one another with respect to the inner product of $L^{2}\left(\mathbb{C}_{+}\right)$(here $p^{\prime}=p /(p-1)$ is the dual exponent). By interpolation theory, then, it follows from the above that

$$
\mathbf{B}^{\downarrow}: L_{q}^{p}\left(\mathbb{C}_{+}\right) \rightarrow L_{q}^{p}\left(\mathbb{C}_{+}\right), \quad \mathbf{B}^{\uparrow}: L_{-q}^{p}\left(\mathbb{C}_{+}\right) \rightarrow L_{-q}^{p}\left(\mathbb{C}_{+}\right),
$$

act boundedly for $0 \leq q \leq p$. This range surely is not best possible, but an understanding of when the Beurling operator $\mathbf{B}$ is bounded in the weighted context (cf., 
e.g., [12]) combined with the approach presented here should lead to the optimal range.

As will be explained later on, the operators $\mathbf{B}^{\uparrow}$ and $\mathbf{B}^{\downarrow}$ are modifications of the Beurling operator $\mathbf{B}$ to the setting of the hyperbolic plane. So it is natural to ask to what extent (1.2) has a hyperbolic analogue. A perhaps naive first try which comes to mind is what are the best constants $B_{1}^{\downarrow}(p), B_{2}^{\downarrow}(p)$ so that

$$
B_{1}^{\downarrow}(p)\|f\|_{L_{p}^{p}\left(\mathbb{C}_{+}\right)} \leq\left\|\mathbf{B}^{\downarrow}[f]\right\|_{L_{p}^{p}\left(\mathbb{C}_{+}\right)} \leq B_{2}^{\downarrow}(p)\|f\|_{L_{p}^{p}\left(\mathbb{C}_{+}\right)}, \quad f \in L_{p}^{p}\left(\mathbb{C}_{+}\right),
$$

It turns out that

$$
\mathbf{B}^{\downarrow}[f]=0, \quad f \in \operatorname{conj}\left(A_{p}^{p}\left(\mathbb{C}_{+}\right)\right),
$$

so that $B_{1}^{\downarrow}(p)=0$ necessarily. While $B_{2}^{\downarrow}(p)$ exists boundedly for all $1<p<+\infty$, the exact value appears to be unknown. For $p=2$ (the Hilbert space case) the methods of this paper give that $B_{2}^{\downarrow}(2) \leq 5$, and they also supply a bound from below; cf. Remark 5.2. If we want a non-trivial two-sided estimate, we need to compare the norm of $\mathbf{B}^{\downarrow}[f]$ with the norm of an expression which vanishes on conj $\left(A_{p}^{p}\left(\mathbb{C}_{+}\right)\right)$. The expression that will work is $\mathbf{V}[f]$, where

$$
\mathbf{V}:=f+4 \mathrm{iEM} .
$$

\section{Commutator identities}

3.1. The commutator of differentiation and multiplication. The commutator of the differentiation operators $\boldsymbol{\partial}, \overline{\boldsymbol{\partial}}$ and the multiplication operator $\mathbf{M}^{n}(n$ is an integer) is given by

$$
\left[\boldsymbol{\partial}, \mathbf{M}^{n}\right]=\boldsymbol{\partial} \mathbf{M}^{n}-\mathbf{M}^{n} \boldsymbol{\partial}=-\frac{\mathrm{i} n}{2} \mathbf{M}^{n-1}, \quad \overline{\boldsymbol{\partial}} \mathbf{M}^{n}-\mathbf{M}^{n} \overline{\boldsymbol{\partial}}=\frac{\mathrm{i} n}{2} \mathbf{M}^{n-1} .
$$

These relations constitute a key step in the proof of the main theorem.

3.2. The commutators of hyperbolic Beurling operators. The various commutators which can be formed using the operators $\mathbf{B}^{\uparrow}, \overline{\mathbf{B}}^{\uparrow}, \mathbf{B}^{\downarrow}, \overline{\mathbf{B}}^{\downarrow}$ can be reduced to one of the following three:

$$
\left[\mathbf{B}^{\downarrow}, \mathbf{B}^{\uparrow}\right], \quad\left[\mathbf{B}^{\downarrow}, \overline{\mathbf{B}}^{\uparrow}\right], \quad\left[\mathbf{B}^{\downarrow}, \overline{\mathbf{B}}^{\downarrow}\right] .
$$

We first consider the commutator

$$
\left[\mathbf{B}^{\downarrow}, \mathbf{B}^{\uparrow}\right]=\mathbf{B}^{\downarrow} \mathbf{B}^{\uparrow}-\mathbf{B}^{\uparrow} \mathbf{B}^{\downarrow} .
$$

To simplify our work, we introduce the compressed Beurling operator $\mathbf{B}_{+}$, which acts contractively on $L^{2}\left(\mathbb{C}_{+}\right)$. It is given by

$$
\mathbf{B}_{+}[f](z):=-\mathrm{pv} \int_{\mathbb{C}_{+}} \frac{f(w)}{(z-w)^{2}} \mathrm{~d} A(w), \quad z \in \mathbb{C}_{+} .
$$

Moreover, we let $\mathbf{P}_{0}$ denote the orthogonal projection $L^{2}\left(\mathbb{C}_{+}\right) \rightarrow A^{2}\left(\mathbb{C}_{+}\right)$, which is given explicitly by

$$
\mathbf{P}_{0}[f](z):=-\int_{\mathbb{C}_{+}} \frac{f(w)}{(z-\bar{w})^{2}} \mathrm{~d} A(w), \quad z \in \mathbb{C}_{+} .
$$

In terms of these operators, we have

$$
\mathbf{B}^{\uparrow}=\mathbf{B}_{+}-\overline{\mathbf{P}}_{0}, \quad \mathbf{B}^{\downarrow}=\mathbf{B}_{+}-\mathbf{P}_{0},
$$


and so

$$
\mathbf{B}^{\downarrow} \mathbf{B}^{\uparrow}=\left(\mathbf{B}_{+}-\mathbf{P}_{0}\right)\left(\mathbf{B}_{+}-\overline{\mathbf{P}}_{0}\right)=\mathbf{B}_{+}^{2}-\mathbf{B}_{+} \overline{\mathbf{P}}_{0}-\mathbf{P}_{0} \mathbf{B}_{+}+\mathbf{P}_{0} \overline{\mathbf{P}}_{0}
$$

while

$$
\mathbf{B}^{\uparrow} \mathbf{B}^{\downarrow}=\left(\mathbf{B}_{+}-\overline{\mathbf{P}}_{0}\right)\left(\mathbf{B}_{+}-\mathbf{P}_{0}\right)=\mathbf{B}_{+}^{2}-\mathbf{B}_{+} \mathbf{P}_{0}-\overline{\mathbf{P}}_{0} \mathbf{B}_{+}+\overline{\mathbf{P}}_{0} \mathbf{P}_{0}
$$

Next, $A^{2}\left(\mathbb{C}_{+}\right)$and conj $\left(A^{2}\left(\mathbb{C}_{+}\right)\right)$are orthogonal to one another in $L^{2}\left(\mathbb{C}_{+}\right)$, and therefore $\mathbf{P}_{0} \overline{\mathbf{P}}_{0}=\overline{\mathbf{P}}_{0} \mathbf{P}_{0}=\mathbf{0}$, where $\mathbf{0}$ stands for the zero operator. For perhaps less obvious reasons (cf. [5]), we also have $\mathbf{P}_{0} \mathbf{B}_{+}=\mathbf{B}_{+} \overline{\mathbf{P}}_{0}=\mathbf{0}$. So, the commutator simplifies significantly:

$$
\left[\mathbf{B}^{\downarrow}, \mathbf{B}^{\uparrow}\right]=\mathbf{B}^{\downarrow} \mathbf{B}^{\uparrow}-\mathbf{B}^{\uparrow} \mathbf{B}^{\downarrow}=\mathbf{B}_{+} \mathbf{P}_{0}+\overline{\mathbf{P}}_{0} \mathbf{B}_{+} .
$$

Put $\mathbf{Q}_{1}:=-2 \mathrm{i} \partial \mathbf{P}_{0}$, which is given explicitly by

$$
\mathbf{Q}_{1}[f](z)=-\int_{\mathbb{C}_{+}} \frac{4 \mathrm{i} f(w)}{(z-\bar{w})^{3}} \mathrm{~d} A(w), \quad z \in \mathbb{C}_{+} .
$$

Then $\mathbf{Q}_{1} \mathbf{M}$ has an interpretation as the orthogonal projection to the holomorphic functions in the $L^{2}$ space over $\mathbb{C}_{+}$with weight $\operatorname{Im} z$. We also see that $\mathbf{Q}_{1}^{*}=\mathbf{Q}_{1}$. Next, a calculation shows that

$$
\int_{\mathbb{C}_{+}} \frac{\mathrm{d} A(\xi)}{(\xi-w)(\xi-\bar{z})^{2}}=\frac{2 \mathrm{i} \operatorname{Im} w}{(\bar{z}-w)^{2}}, \quad z, w \in \mathbb{C}_{+},
$$

and differentiation with respect to $w$ on both sides yields

$$
\operatorname{pv} \int_{\mathbb{C}_{+}} \frac{\mathrm{d} A(\xi)}{(\xi-w)^{2}(\xi-\bar{z})^{2}}=\boldsymbol{\partial}_{w}\left\{\frac{2 \mathrm{i} \operatorname{Im} w}{(\bar{z}-w)^{2}}\right\}=\frac{1}{(\bar{z}-w)^{2}}+\frac{4 \mathrm{i} \operatorname{Im} w}{(\bar{z}-w)^{3}}, \quad z, w \in \mathbb{C}_{+} .
$$

This leads to the operator identities

$$
\overline{\mathbf{P}}_{0} \mathbf{B}_{+}=-\overline{\mathbf{P}}_{0}+\overline{\mathbf{Q}}_{1} \mathbf{M}, \quad \mathbf{B}_{+} \mathbf{P}_{0}=-\mathbf{P}_{0}+\mathbf{M} \mathbf{Q}_{1}
$$

and so

$$
\left[\mathbf{B}^{\downarrow}, \mathbf{B}^{\uparrow}\right]=-\mathbf{P}_{0}-\overline{\mathbf{P}}_{0}+\overline{\mathbf{Q}}_{1} \mathbf{M}+\mathbf{M Q}_{1} .
$$

The second commutator to be considered is

$$
\left[\mathbf{B}^{\downarrow}, \overline{\mathbf{B}}^{\uparrow}\right]=\mathbf{B}^{\downarrow} \overline{\mathbf{B}}^{\uparrow}-\overline{\mathbf{B}}^{\uparrow} \mathbf{B}^{\downarrow} .
$$

If we use (3.3) and follow along the lines of the preceding commutator calculation, we find that

$$
\left[\mathbf{B}^{\downarrow}, \overline{\mathbf{B}}^{\uparrow}\right]=\left[\mathbf{B}_{+}, \overline{\mathbf{B}}_{+}\right]-\mathbf{P}_{0} \overline{\mathbf{B}}_{+}-\mathbf{B}_{+} \mathbf{P}_{0}=\left[\mathbf{B}_{+}, \overline{\mathbf{B}}_{+}\right]+2 \mathbf{P}_{0}-\mathbf{M Q}_{1}-\mathbf{Q}_{1} \mathbf{M} .
$$

We readily find that

$$
\overline{\mathbf{B}}_{+} \mathbf{B}_{+}=\mathbf{I}-\overline{\mathbf{P}}_{0}, \quad \mathbf{B}_{+} \overline{\mathbf{B}}_{+}=\mathbf{I}-\mathbf{P}_{0},
$$

where $\mathbf{I}$ is the identity operator, so that

$$
\left[\mathbf{B}_{+}, \overline{\mathbf{B}}_{+}\right]=\overline{\mathbf{P}}_{0}-\mathbf{P}_{0}
$$

and consequently

$$
\left[\mathbf{B}^{\downarrow}, \overline{\mathbf{B}}^{\uparrow}\right]=\overline{\mathbf{P}}_{0}+\mathbf{P}_{0}-\mathbf{M Q}_{1}-\mathrm{Q}_{1} \mathbf{M} .
$$


This commutator relation is interesting because it leads to the following norm identity:

$$
\begin{aligned}
& \left\|\overline{\mathbf{B}}^{\uparrow}[f]\right\|_{L^{2}\left(\mathbb{C}_{+}\right)}^{2}-\left\|\mathbf{B}^{\downarrow}[f]\right\|_{L^{2}\left(\mathbb{C}_{+}\right)}^{2} \\
& =\left\|\overline{\mathbf{P}}_{0}[f]\right\|_{L^{2}\left(\mathbb{C}_{+}\right)}^{2}+\left\|\left(\mathbf{P}_{0}-\mathbf{Q}_{1} \mathbf{M}\right)[f]\right\|_{L^{2}\left(\mathbb{C}_{+}\right)}^{2}-\left\|\mathbf{Q}_{1} \mathbf{M}[f]\right\|_{L^{2}\left(\mathbb{C}_{+}\right)}^{2} .
\end{aligned}
$$

The third commutator is

$$
\left[\mathbf{B}^{\downarrow}, \overline{\mathbf{B}}^{\downarrow}\right]=\mathbf{B}^{\downarrow} \overline{\mathbf{B}}^{\downarrow}-\overline{\mathbf{B}}^{\downarrow} \mathbf{B}^{\downarrow} .
$$

The method employed above yields that

$$
\left[\mathbf{B}^{\downarrow}, \overline{\mathbf{B}}^{\downarrow}\right]=\overline{\mathbf{P}}_{0}-\mathrm{MQ}_{1},
$$

and, as a consequence, we may also derive that

$$
\left[\mathbf{B}^{\uparrow}, \overline{\mathbf{B}}^{\uparrow}\right]=\overline{\mathbf{P}}_{0}-\mathbf{Q}_{1} \mathbf{M} \text {. }
$$

\section{A hyperbolic Liouville-type theorem}

4.1. A Liouville-type theorem for the hyperbolic plane. By Liouville's theorem, the only bounded harmonic functions in the complex plane are the constants. If we ask the functions to be in $L^{p}(\mathbb{C})$ as well, the only harmonic function is the constant 0 . A hyperbolic plane analogue of this statement is offered by the following (see [8] for details).

Theorem 4.1. (Hedenmalm, Parissis, Saksman) Suppose a function $f \in L_{q}^{p}\left(\mathbb{C}_{+}\right)$ is harmonic in $\mathbb{C}_{+}$, where $q$ is real and $1 \leq p<+\infty$. If $q \leq-1$, then $f=0$. On the other hand, if $-1<q$, there are nontrivial harmonic functions $f$ in $L_{q}^{p}\left(\mathbb{C}_{+}\right)$.

Remark 4.2. (a) For $0<p<1$ and $q \leq-2$ the theorem follows from Suzuki [13].

4.2. A biharmonic Liouville-type theorem for the hyperbolic plane. A function $f$ with $\Delta^{2} f=0$ is said to be biharmonic. The biharmonic functions in $\mathbb{C}_{+}$ all have the form $f=f_{1}+\mathbf{M} f_{2}$, where $f_{1}, f_{2}$ are both harmonic in $\mathbb{C}_{+}$.

Theorem 4.3. (Hedenmalm, Parissis, Saksman) Suppose a function $f \in L_{q}^{p}\left(\mathbb{C}_{+}\right)$ is biharmonic in $\mathbb{C}_{+}$, where $-\infty<q \leq-1$ and $1 \leq p<+\infty$. Then $\mathbf{M}^{-1} f$ is harmonic in $\mathbb{C}_{+}$.

\section{Main results}

5.1. The norm estimate of the hyperbolic plane Beurling transform. We now state our main theorem.

Theorem 5.1. $(1<p<+\infty)$ The operators

$$
\mathbf{B}^{\uparrow}: L_{-p}^{p}\left(\mathbb{C}_{+}\right) \rightarrow L_{-p}^{p}\left(\mathbb{C}_{+}\right), \quad \mathbf{B}^{\downarrow}: L_{p}^{p}\left(\mathbb{C}_{+}\right) \rightarrow L_{p}^{p}\left(\mathbb{C}_{+}\right),
$$

are bounded. Indeed, in terms of the operator $\mathbf{V}=\mathbf{I}+4 \mathbf{i E M}$, we have the norm estimate

$$
B(p)^{-1}\|\mathbf{V}[f]\|_{L_{p}^{p}\left(\mathbb{C}_{+}\right)} \leq\left\|\mathbf{B}^{\downarrow}[f]\right\|_{L_{p}^{p}\left(\mathbb{C}_{+}\right)} \leq B(p)\|\mathbf{V}[f]\|_{L_{p}^{p}\left(\mathbb{C}_{+}\right)}
$$

for all $f \in L_{p}^{p}\left(\mathbb{C}_{+}\right)$. Here, $B(p)$ denotes the norm of the Beurling transform on $L^{p}(\mathbb{C})$, and the constants are best possible on both sides of the estimate. 
Proof. As we saw in Subsection 1.7, it is a consequence of the Hardy inequality (1.6) that $\mathbf{C}^{\downarrow}[f]$ and $\overline{\mathbf{C}}^{\downarrow}[f]$ are in $L^{p}\left(\mathbb{C}_{+}\right)$provided that $f \in L_{p}^{p}\left(\mathbb{C}_{+}\right)$.

Next, we shall assume $f \in L_{p}^{p}\left(\mathbb{C}_{+}\right)$is of the form $f=\Delta F$, for some $F \in C_{c}^{\infty}\left(\mathbb{C}_{+}\right)$. We first claim that the collection of such $f$ is dense in $L_{p}^{p}\left(\mathbb{C}_{+}\right)$. To this end, suppose $g \in L^{p^{\prime}}\left(\mathbb{C}_{+}\right)\left(p^{\prime}=p /(p-1)\right.$ is the dual exponent $)$ is such that

$$
\int_{\mathbb{C}_{+}}(\operatorname{Im} z) \Delta F(z) \bar{g}(z) \mathrm{d} A(z)=0 .
$$

By Green's formula, we get, in the sense of distribution theory,

$$
\int_{\mathbb{C}_{+}} F(z) \boldsymbol{\Delta}((\operatorname{Im} z) \bar{g}(z)) \mathrm{d} A(z)=0,
$$

for all $F \in C_{c}^{\infty}\left(\mathbb{C}_{+}\right)$. It follows that

$$
\Delta \mathbf{M}[g]=0,
$$

so that $\mathbf{M}[g] \in L_{-p^{\prime}}^{p^{\prime}}\left(\mathbb{C}_{+}\right)$is harmonic. By Theorem 4.1, we get $g=0$, and the claim follows.

In terms of the function $F$, we have

$$
\mathbf{C}^{\downarrow}[f]=\boldsymbol{\partial} F, \quad \overline{\mathbf{C}}^{\downarrow}[f]=\bar{\partial} F .
$$

This is so because of Havin's lemma. We now see that the norm estimate of the theorem follows once it has been established that

$$
\begin{aligned}
& B(p)^{-1}\left\|\mathbf{M} \boldsymbol{\Delta} F+\frac{\mathrm{i}}{2}(\boldsymbol{\partial} F+\overline{\boldsymbol{\partial}} F)\right\|_{L^{p}\left(\mathbb{C}_{+}\right)} \\
& \leq\left\|\mathbf{M} \boldsymbol{\partial}^{2} F\right\|_{L^{p}\left(\mathbb{C}_{+}\right)} \leq B(p)\left\|\mathbf{M} \boldsymbol{\Delta} F+\frac{\mathrm{i}}{2}(\boldsymbol{\partial} F+\overline{\boldsymbol{\partial}} F)\right\|_{L^{p}\left(\mathbb{C}_{+}\right)} .
\end{aligned}
$$

We know that for $G \in C_{c}^{\infty}\left(\mathbb{C}_{+}\right)$,

$$
\frac{1}{B(p)}\|\bar{\partial} G\|_{L^{p}\left(\mathbb{C}_{+}\right)} \leq\|\boldsymbol{\partial} G\|_{L^{p}\left(\mathbb{C}_{+}\right)} \leq B(p)\|\bar{\partial} G\|_{L^{p}(\mathbb{C})_{+}}
$$

This is a consequence of $(1.3)$, as $G$ extends to a function in $C_{c}^{\infty}(\mathbb{C})$ if we declare it to vanish on $\mathbb{C} \backslash \mathbb{C}_{+}$. So we find ourselves looking for a $G$ with $\partial G=\mathbf{M} \partial^{2} F$. Now, in view of the commutator identity (3.1),

$$
\mathbf{M} \partial^{2}=\boldsymbol{\partial}\left(\mathbf{M} \boldsymbol{\partial}+\frac{\mathrm{i}}{2} \mathbf{I}\right)
$$

so that

$$
G:=\left(\mathbf{M} \boldsymbol{\partial}+\frac{\mathrm{i}}{2} \mathbf{I}\right)[F] \in C_{c}^{\infty}\left(\mathbb{C}_{+}\right)
$$

is the obvious candidate. We calculate that

$$
\overline{\boldsymbol{\partial}} G=\overline{\boldsymbol{\partial}}\left(\mathbf{M} \boldsymbol{\partial}+\frac{\mathrm{i}}{2} \mathbf{I}\right)[F]=\left(\mathbf{M} \boldsymbol{\Delta}+\frac{\mathrm{i}}{2} \boldsymbol{\partial}+\frac{\mathrm{i}}{2} \overline{\boldsymbol{\partial}}\right)[F]
$$

if we use again (3.1) and recall that $\boldsymbol{\Delta}=\overline{\boldsymbol{\partial}} \boldsymbol{\partial}$. The claim (5.1) is now an immediate consequence of (5.2). The optimality of the constants will be discussed in Subsection 7.3. Except for that point, the proof is complete.

Remark 5.2. For $p=2$, we get a norm equality in Theorem 5.1. So, to analyze the norm of $\mathbf{B}^{\downarrow}$ on $L_{2}^{2}\left(\mathbb{C}_{+}\right)$, we might as well analyze the norm of $\mathbf{V}$. By the triangle inequality and Subsection 1.9, we get

$$
\|\mathbf{V}\| \leq\|\mathbf{I}\|+4\|\mathbf{E M}\| \leq 5
$$


where the norm is as an operator on $L_{2}^{2}\left(\mathbb{C}_{+}\right)$. Next, if $f \in L_{2}^{2}\left(\mathbb{C}_{+}\right)$is real-valued, then

$$
\|\mathbf{V}[f]\|_{L_{2}^{2}\left(\mathbb{C}_{+}\right)}^{2}=\|f\|_{L_{2}^{2}\left(\mathbb{C}_{+}\right)}^{2}+16\|\mathbf{E M}[f]\|_{L_{2}^{2}\left(\mathbb{C}_{+}\right)}^{2},
$$

so by optimizing over such real-valued functions, we obtain the reverse inequality

$$
\|\mathbf{V}\| \geq \sqrt{1+16\|\mathbf{E M}\|^{2}}
$$

\section{The analysis of two operators}

6.1. The operators. In the context of Theorem 5.1, with $p=2$, we would like to study the operators

$$
\mathbf{B}^{\downarrow}: L_{2}^{2}\left(\mathbb{C}_{+}\right) \rightarrow L_{2}^{2}\left(\mathbb{C}_{+}\right)
$$

and

$$
\mathbf{V}=\mathbf{I}+4 \mathrm{iEM}: L_{2}^{2}\left(\mathbb{C}_{+}\right) \rightarrow L_{2}^{2}\left(\mathbb{C}_{+}\right)
$$

with respect to range and null space ( $\mathbf{I}$ is the identity operator). It is a curious fact that this problem - for the second operator - is intimately connected with the classical Whittaker (or Kummer) ordinary differential equation (see, e.g., [1], [10], or Wolfram MathWorld, Wikipedia). In view of Theorem 5.1, the null spaces of the two operators coincide, which is why we only characterize the null space of the second operator.

6.2. The range of the operator $\mathbf{B}^{\downarrow}$. The range of $\mathbf{M B}^{\downarrow}$ is a subspace of $L^{2}\left(\mathbb{C}_{+}\right)$, and studying the range of $\mathbf{M B}^{\downarrow}$ is equivalent to studying the range of $\mathbf{B}^{\downarrow}$. Let $h \in L_{-2}^{2}\left(\mathbb{C}_{+}\right)$be such that $\mathbf{M}^{-1}[h] \in L^{2}\left(\mathbb{C}_{+}\right)$is perpendicular to the range of MB $^{\downarrow}$. From the proof of Theorem 5.1, we see that this is the same as requiring that

$$
\left\langle\mathbf{M}^{-1}[h], \mathbf{M} \partial^{2} F\right\rangle_{L^{2}\left(\mathbb{C}_{+}\right)}=0, \quad F \in C_{c}^{\infty}\left(\mathbb{C}_{+}\right) .
$$

By dualizing, we see that this is the same as

$$
\left\langle\overline{\boldsymbol{\partial}}^{2} h, F\right\rangle_{L^{2}\left(\mathbb{C}_{+}\right)}=0, \quad F \in C_{c}^{\infty}\left(\mathbb{C}_{+}\right),
$$

that is,

$$
\bar{\partial}^{2} h=0 .
$$

This means that $h$ is bi-analytic in $\mathbb{C}_{+}$, and hence of the form $h=h_{1}+\bar{z} h_{2}$, where $h_{1}, h_{2}$ are analytic in $\mathbb{C}_{+}$. We rewrite this decomposition in the form $h=h_{3}+\mathbf{M} h_{4}$, where $h_{3}, h_{4}$ are analytic; simply put $h_{3}:=h_{1}+z h_{2}$ and $h_{4}:=-2 \mathrm{i} h_{2}$. In particular, $h$ is biharmonic, and since $h \in L_{-2}^{2}\left(\mathbb{C}_{+}\right)$, Theorem 4.3 gives that $h_{3}=0$, so that $h=\mathbf{M} h_{4}$ where $h_{4} \in A^{2}\left(\mathbb{C}_{+}\right)$. We conclude that the $L^{2}\left(\mathbb{C}_{+}\right)$-closure of the range of $\mathbf{M B}^{\downarrow}$ equals $L^{2}\left(\mathbb{C}_{+}\right) \ominus A^{2}\left(\mathbb{C}_{+}\right)$, so that the $L_{2}^{2}\left(\mathbb{C}_{+}\right)$-closure of the range of $\mathbf{B}^{\downarrow}$ is equal to

$$
\mathbf{M}^{-1}\left[L^{2}\left(\mathbb{C}_{+}\right) \ominus A^{2}\left(\mathbb{C}_{+}\right)\right] .
$$

6.3. The range of the operator $\mathbf{V}$. Let $h \in L_{-2}^{2}\left(\mathbb{C}_{+}\right)$be such that $\mathbf{M}^{-1}[h] \in$ $L^{2}\left(\mathbb{C}_{+}\right)$is perpendicular to the range of the above operator. From the proof of Theorem 5.1, we see that this is the same as requiring that

$$
\left\langle\mathbf{M}^{-1}[h], \mathbf{M} \boldsymbol{\Delta} F+\frac{\mathrm{i}}{2}(\boldsymbol{\partial}+\overline{\boldsymbol{\partial}}) F\right\rangle_{L^{2}\left(\mathbb{C}_{+}\right)}=0, \quad F \in C_{c}^{\infty}\left(\mathbb{C}_{+}\right) .
$$

By dualizing we see that this is the same as

$$
\left\langle\boldsymbol{\Delta} h+\frac{\mathrm{i}}{2}(\boldsymbol{\partial}+\overline{\boldsymbol{\partial}}) \mathbf{M}^{-1}[h], F\right\rangle_{L^{2}\left(\mathbb{C}_{+}\right)}=0, \quad F \in C_{c}^{\infty}\left(\mathbb{C}_{+}\right),
$$


that is,

$$
\Delta h+\frac{\mathrm{i}}{2}(\boldsymbol{\partial}+\overline{\boldsymbol{\partial}}) \mathbf{M}^{-1}[h]=0 .
$$

Since

this amounts to the differential equation

$$
\partial+\bar{\partial}=\frac{\partial}{\partial x}
$$

$$
y\left(\frac{\partial^{2}}{\partial x^{2}}+\frac{\partial^{2}}{\partial y^{2}}\right) h+2 \mathrm{i} \frac{\partial}{\partial x} h=0 .
$$

Lemma 6.1. A function $h \in L_{-2}^{2}\left(\mathbb{C}_{+}\right)$solves the partial differential equation (6.1) in $\mathbb{C}_{+}$if and only if $\mathbf{M}^{-1}[h] \in \operatorname{conj}\left(A^{2}\left(\mathbb{C}_{+}\right)\right)$.

Proof. Let

$$
\mathbf{F}_{1}[h](\xi, y)=\int_{-\infty}^{+\infty} \mathrm{e}^{-\mathrm{i} x \xi} h(x, y) \mathrm{d} x
$$

denote the partial Fourier transform with respect to the $x$ variable. Here, we use the standard identification of $\mathbb{C} \cong \mathbb{R}^{2}$, so that $h(x, y)=h(x+\mathrm{i} y)$. An application of the partial Fourier transform to the differential equation (6.1) yields

$$
y\left(-\xi^{2}+\frac{\partial^{2}}{\partial y^{2}}\right) \mathbf{F}_{1}[h](\xi, y)-2 \xi \mathbf{F}_{1}[h](\xi, y)=0
$$

that is,

$$
\frac{\partial^{2}}{\partial y^{2}} \mathbf{F}_{1}[h](\xi, y)-\left(\xi^{2}+\frac{2 \xi}{y}\right) \mathbf{F}_{1}[h](\xi, y)=0 .
$$

Next, we put

$$
H(\xi, t):=\mathbf{F}_{1}[h]\left(\xi, \frac{t}{2|\xi|}\right),
$$

and see that (6.2) becomes

$$
\frac{\partial^{2}}{\partial t^{2}} H(\xi, t)-\left(\frac{1}{4}+\frac{\operatorname{sgn}(\xi)}{t}\right) H(\xi, t)=0 .
$$

The requirement that $h \in L_{-2}^{2}\left(\mathbb{C}_{+}\right)$amounts to

$$
\int_{0}^{+\infty} \int_{-\infty}^{+\infty}|H(\xi, t)|^{2} \frac{|\xi| \mathrm{d} \xi \mathrm{d} t}{t^{2}}<+\infty
$$

The differential equation (6.3) is of Whittaker type. It is well-known that the general solution to the ordinary differential equation

$$
\frac{d^{2}}{\partial t^{2}} X(t)-\left(\frac{1}{4}+\frac{1}{t}\right) X(t)=0
$$

is of the form

$$
X(t)=A_{1} t \mathrm{e}^{t / 2}+B_{1} t \mathrm{e}^{-t / 2} \int_{0}^{+\infty} \mathrm{e}^{-t \theta} \frac{\theta}{1+\theta} \mathrm{d} \theta
$$

where $A_{1}, B_{1}$ are constants, while the general solution to the ordinary differential equation

$$
\frac{d^{2}}{\partial t^{2}} Y(t)-\left(\frac{1}{4}-\frac{1}{t}\right) Y(t)=0
$$


is of the form

$$
Y(t)=A_{2} \mathrm{e}^{-t / 2}\left(1-t \log t-t \int_{0}^{t} \frac{\mathrm{e}^{\theta}-1-\theta}{\theta^{2}} \mathrm{~d} \theta\right)+B_{2} t \mathrm{e}^{-t / 2},
$$

where $A_{2}, B_{2}$ are constants. It follows that $H(\xi, t)$ must have form

$$
H(\xi, t)=A_{1}(\xi) t \mathrm{e}^{t / 2}+B_{1}(\xi) t \mathrm{e}^{-t / 2} \int_{0}^{+\infty} \mathrm{e}^{-t \theta} \frac{\theta}{1+\theta} \mathrm{d} \theta, \quad \xi>0,
$$

and

$$
H(\xi, t)=A_{2}(\xi) \mathrm{e}^{-t / 2}\left(1-t \log t-t \int_{0}^{t} \frac{\mathrm{e}^{\theta}-1-\theta}{\theta^{2}} \mathrm{~d} \theta\right)+B_{2}(\xi) t \mathrm{e}^{-t / 2}, \quad \xi<0 .
$$

A careful analysis of the behavior of these solutions as $t \rightarrow 0^{+}$and $t \rightarrow+\infty$ shows that (6.4) is impossible unless $A_{1}(\xi)=B_{1}(\xi)=A_{2}(\xi)=0$, in which case

$$
H(\xi, t)=0, \quad \xi>0,
$$

and

$$
H(\xi, t)=B_{2}(\xi) t \mathrm{e}^{-t / 2}, \quad \xi<0 .
$$

The function $B_{2}(\xi)$ must then satisfy

$$
\int_{-\infty}^{0}|\xi|\left|B_{2}(\xi)\right|^{2} \mathrm{~d} \xi<+\infty
$$

and the partial Fourier transform $\widehat{h}$ takes the form

$$
\mathbf{F}_{1}[h](\xi, y)=2|\xi| y B_{2}(\xi) \mathrm{e}^{y \xi} 1_{]-\infty, 0]}(\xi),
$$

so that

$$
\mathbf{F}_{1}\left[\mathbf{M}^{-1}[h]\right](\xi, y)=B_{3}(\xi) \mathrm{e}^{y \xi} 1_{]-\infty, 0]}(\xi),
$$

where $B_{3}(\xi):=2|\xi| B_{2}(\xi)$. This form of $\widehat{h}$ is equivalent to the assertion that $\mathbf{M}^{-1}[h] \in$ $\operatorname{conj}\left(A^{2}\left(\mathbb{C}_{+}\right)\right)$. This follows from the following two observations: $(i)$ the support of its partial Fourier transform is contained in ] $-\infty, 0]$, and $(i i)$ the exponential factor $\mathrm{e}^{-y|\xi|}$ is the Fourier transform of the Poisson kernel.

We now obtain the closure of the range of the operator.

Proposition 6.2. The closure of the range of $\mathbf{V}=\mathbf{I}+4 \mathrm{iEM}: L_{2}^{2}\left(\mathbb{C}_{+}\right) \rightarrow L_{2}^{2}\left(\mathbb{C}_{+}\right)$ equals

$$
\mathbf{M}\left[L^{2}\left(\mathbb{C}_{+}\right) \ominus \operatorname{conj}\left(A^{2}\left(\mathbb{C}_{+}\right)\right)\right]
$$

Remark 6.3. One can show that the range of the operator is closed.

The null space of the operator. We turn to the study of the null space of the operator $\mathbf{V}$, which is the same as the null space of $\mathbf{B}^{\downarrow}$. So, given $f \in L_{2}^{2}\left(\mathbb{C}_{+}\right)$, we want to know what the solutions to

$$
\mathbf{M V}[f]=\mathbf{M}[f]+4 \operatorname{iMEM}[f]=0
$$

look like. Let $F \in L_{-2}^{2}\left(\mathbb{C}_{+}\right)$be the associated function

$$
F=\overline{\mathbf{C}}^{\uparrow} \mathbf{C}^{\downarrow}[f]=\mathbf{C}^{\uparrow} \overline{\mathbf{C}}^{\downarrow}[f] .
$$

Then $\Delta F=f$, and (6.5) takes the form

$$
\mathbf{M}[\boldsymbol{\Delta} F]+\frac{\mathrm{i}}{2}[\boldsymbol{\partial} F+\overline{\boldsymbol{\partial}} F]=0 .
$$


By Lemma 6.1, we find that this happens if and only if $\mathbf{M}^{-1}[F] \in \operatorname{conj}\left(A^{2}\left(\mathbb{C}_{+}\right)\right)$. From this, we quickly derive the following characterization of the null space.

Proposition 6.4. The null space of the operator $\mathbf{V}=\mathbf{I}+4 \mathrm{iEM}: L_{2}^{2}\left(\mathbb{C}_{+}\right) \rightarrow$ $L^{2}\left(\mathbb{C}_{+}\right)$is equal to $\operatorname{conj}\left(A_{2}^{2}\left(\mathbb{C}_{+}\right)\right)$.

\section{Geometric interpretation of the main theorem}

7.1. The hyperbolic plane and differential operators. Associated with the half-plane model of the hyperbolic plane $\mathbb{H}$, we have the geometrically induced differential operators $\partial^{\uparrow}, \bar{\partial}^{\uparrow}$ :

$$
\partial^{\uparrow}=\mathrm{M} \partial, \quad \bar{\partial}^{\uparrow}=\mathrm{M} \bar{\partial} .
$$

After all, the length scale on $\mathbb{C}_{+}$should be modified to correspond to that of the hyperbolic plane. There are also the "dual" geometrically induced differential operators $\partial^{\downarrow}, \bar{\partial}^{\downarrow}:$

$$
\partial^{\downarrow}=\mathbf{M}^{2} \partial \mathbf{M}^{-1}, \quad \bar{\partial}^{\downarrow}=\mathbf{M}^{2} \bar{\partial} \mathbf{M}^{-1}
$$

with the properties that

$$
\left\langle\boldsymbol{\partial}^{\uparrow} f, g\right\rangle_{L^{2}\left(\mathbb{C}_{+}\right)}=-\left\langle f, \overline{\boldsymbol{\partial}}^{\downarrow} g\right\rangle_{L^{2}\left(\mathbb{C}_{+}\right)}, \quad\left\langle\overline{\boldsymbol{\partial}}^{\uparrow} f, g\right\rangle_{L^{2}\left(\mathbb{C}_{+}\right)}=-\left\langle f, \boldsymbol{\partial}^{\downarrow} g\right\rangle_{L^{2}\left(\mathbb{C}_{+}\right)},
$$

provided at least one of $f, g$ is in the class $C_{c}^{\infty}\left(\mathbb{C}_{+}\right)$of compactly supported test functions, and the other is, say, locally integrable on $\mathbb{C}_{+}$(the partial derivatives are interpreted in the sense of distribution theory when necessary). The hyperbolic Laplacian $\boldsymbol{\Delta}_{\mathbb{H}}$ is obtained as a combination of two such geometric differential operators:

$$
\Delta_{\mathbb{H}}=\bar{\partial}^{\downarrow} \partial^{\uparrow}=\partial^{\downarrow} \bar{\partial}^{\uparrow}=\mathrm{M}^{2} \Delta .
$$

7.2. Hyperbolic differential operators and the hyperbolic plane Beurling operators. In analogy with the planar Beurling transform $\mathbf{B}=\boldsymbol{\partial} \overline{\boldsymbol{\partial}}^{-1}$, a natural candidate for the "hyperbolic plane Beurling transform" is $\boldsymbol{\partial}^{\downarrow}\left(\overline{\boldsymbol{\partial}}^{\downarrow}\right)^{-1}$. Like in the case of the Euclidean plane, there is the matter of the choice of $\left(\bar{\partial}^{\downarrow}\right)^{-1}$. In contrast with the $\bar{\partial}$-problem in the plane, given a function $f \in L^{2}(\mathbb{H}):=L_{-2}^{2}\left(\mathbb{C}_{+}\right)$, there always exists a solution $u \in L^{2}(\mathbb{H})$ with $\bar{\partial}^{\downarrow} u=f$, and

$$
\|u\|_{L^{2}(\mathbb{H})} \leq 4\|f\|_{L^{2}(\mathbb{H})} .
$$

This follows from the Hardy inequality in a manner which was explained in Subsections 1.6 and 1.7. In particular, there always exists a unique solution $u=u_{f}$ of minimal norm in $L_{-2}^{2}\left(\mathbb{C}_{+}\right)$. We write $u_{f}=\left[\overline{\boldsymbol{\partial}}^{\downarrow}\right]_{\min }^{-1} f$ for this minimal solution, and have thus defined the linear operator $\left[\overline{\boldsymbol{\partial}}^{\downarrow}\right]_{\min }^{-1}$. In a similar manner, we may define the operator $\left[\boldsymbol{\partial}^{\downarrow}\right]_{\min }^{-1}$. It is easy to see that

$$
\left[\overline{\boldsymbol{\partial}}^{\downarrow}\right]_{\min }^{-1}=\mathbf{M} \mathbf{C}^{\downarrow} \mathbf{M}^{-2}, \quad\left[\boldsymbol{\partial}^{\downarrow}\right]_{\min }^{-1}=\mathbf{M} \overline{\mathbf{C}}^{\downarrow} \mathbf{M}^{-2},
$$

and hence $\left[\boldsymbol{\partial}^{\downarrow}\right]_{\text {min }}^{-1},\left[\overline{\boldsymbol{\partial}}^{\downarrow}\right]_{\text {min }}^{-1}$ both act boundedly on $L_{-p}^{p}\left(\mathbb{C}_{+}\right)$for $1<p<+\infty$. Moreover, since

$$
\boldsymbol{\partial}^{\downarrow}\left[\overline{\boldsymbol{\partial}}^{\downarrow}\right]_{\min }^{-1}=\left(\mathbf{M}^{2} \boldsymbol{\partial} \mathbf{M}^{-1}\right)\left(\mathbf{M} \mathbf{C}^{\downarrow} \mathbf{M}^{-2}\right)=\mathbf{M}^{2} \boldsymbol{\partial} \mathbf{C}^{\downarrow} \mathbf{M}^{-2}=\mathbf{M}^{2} \mathbf{B}^{\downarrow} \mathbf{M}^{-2},
$$

we see that the operator $\mathbf{B}^{\downarrow}$ is indeed a Beurling-type operator associated with the hyperbolic plane. Theorem 5.1 may now be formulated in these geometric-differential operator terms. 
Theorem 7.1. $(1<p<+\infty)$ For $p=2$, we have the norm identity

$$
\left\|\boldsymbol{\partial}^{\downarrow}\left[\overline{\boldsymbol{\partial}}^{\downarrow}\right]_{\min }^{-1} f\right\|_{L_{-2}^{2}\left(\mathbb{C}_{+}\right)}=\left\|f+\frac{\mathrm{i}}{2}\left(\left[\overline{\boldsymbol{\partial}}^{\downarrow}\right]_{\min }^{-1} f+\left[\boldsymbol{\partial}^{\downarrow}\right]_{\min }^{-1} f\right)\right\|_{L_{-2}^{2}\left(\mathbb{C}_{+}\right)}, \quad f \in L_{-2}^{2}\left(\mathbb{C}_{+}\right),
$$

while for general $p$, we have

$$
\begin{aligned}
& \frac{1}{B(p)}\left\|f+\frac{\mathrm{i}}{2}\left(\left[\overline{\boldsymbol{\partial}}^{\downarrow}\right]_{\min }^{-1} f+\left[\boldsymbol{\partial}^{\downarrow}\right]_{\min }^{-1} f\right)\right\|_{L_{-p}^{p}\left(\mathbb{C}_{+}\right)} \leq\left\|\boldsymbol{\partial}^{\downarrow}\left[\overline{\boldsymbol{\partial}}^{\downarrow}\right]_{\min }^{-1} f\right\|_{L_{-p}^{p}\left(\mathbb{C}_{+}\right)} \\
& \leq B(p)\left\|f+\frac{\mathrm{i}}{2}\left(\left[\overline{\boldsymbol{\partial}}^{\downarrow}\right]_{\min }^{-1} f+\left[\boldsymbol{\partial}^{\downarrow}\right]_{\min }^{-1} f\right)\right\|_{L_{-p}^{p}\left(\mathbb{C}_{+}\right)}, \quad f \in L_{-p}^{p}\left(\mathbb{C}_{+}\right) .
\end{aligned}
$$

Here, $B(p)$ is the norm of $\mathbf{B}: L^{p}(\mathbb{C}) \rightarrow L^{p}(\mathbb{C})$. The indicated constants are optimal.

7.3. Explanation of the sharpness of the constants. Let introduce, for real $\alpha \geq 0$, the half-plane

$$
\mathbb{C}_{+}^{\alpha}=\{z \in \mathbb{C}: \operatorname{Im} z>-\alpha\},
$$

supplied with the metric and area measure

$$
\mathrm{d} s_{\alpha}(z)=\frac{(1+\alpha)|\mathrm{d} z|}{\alpha+\operatorname{Im} z}, \quad \mathrm{~d} A_{\alpha}(z)=\frac{(1+\alpha)^{2} \mathrm{~d} A(z)}{(\alpha+\operatorname{Im} z)^{2}}
$$

and write

$$
\mathbb{H}^{\alpha}=\left\langle\mathbb{C}_{+}^{\alpha}, \mathrm{d} s_{\alpha}\right\rangle
$$

for this model of the hyperbolic plane. For $\alpha=0$ we get the standard model of the hyperbolic plane, while as $\alpha \rightarrow+\infty$ the model (locally) flattens out to give the Euclidean plane in the limit. We consider the associated multiplication operator

$$
\mathbf{M}_{\alpha}[f](z)=\frac{\operatorname{Im} z+\alpha}{1+\alpha} f(z), \quad z \in \mathbb{C}_{+}^{\alpha},
$$

and the differential operators

$$
\partial_{\alpha}^{\uparrow}=\mathbf{M}_{\alpha} \partial, \quad \overline{\boldsymbol{\partial}}_{\alpha}^{\uparrow}=\mathbf{M}_{\alpha} \overline{\boldsymbol{\partial}}, \quad \partial_{\alpha}^{\downarrow}=\mathbf{M}_{\alpha}^{2} \partial \mathbf{M}_{\alpha}^{-1}, \quad \overline{\boldsymbol{\partial}}_{\alpha}^{\downarrow}=\mathbf{M}_{\alpha}^{2} \overline{\boldsymbol{\partial}} \mathbf{M}_{\alpha}^{-1} .
$$

The estimate of Theorem 7.1 for general $1<p<+\infty$ now takes the form (with obvious notation)

$$
\begin{aligned}
& \frac{1}{B(p)}\left\|f+\frac{\mathrm{i}}{2(1+\alpha)}\left(\left[\overline{\boldsymbol{\partial}}_{\alpha}^{\downarrow}\right]_{\text {min }}^{-1} f+\left[\boldsymbol{\partial}_{\alpha}^{\downarrow}\right]_{\min }^{-1} f\right)\right\|_{L_{-p}^{p}\left(\mathbb{C}_{+}^{\alpha}\right)} \leq\left\|\boldsymbol{\partial}_{\alpha}^{\downarrow}\left[\overline{\boldsymbol{\partial}}_{\alpha}^{\downarrow}\right]_{\min }^{-1} f\right\|_{L_{-p}^{p}\left(\mathbb{C}_{+}^{\alpha}\right)} \\
& \leq B(p)\left\|f+\frac{\mathrm{i}}{2(1+\alpha)}\left(\left[\overline{\boldsymbol{\partial}}_{\alpha}^{\downarrow}\right]_{\min }^{-1} f+\left[\boldsymbol{\partial}_{\alpha}^{\downarrow}\right]_{\min }^{-1} f\right)\right\|_{L_{-p}^{p}\left(\mathbb{C}_{+}^{\alpha}\right)}, \quad f \in L_{-p}^{p}\left(\mathbb{C}_{+}^{\alpha}\right) .
\end{aligned}
$$

As $\alpha \rightarrow+\infty$, the geometry becomes eventually Euclidean, and the estimate becomes

$$
\frac{1}{B(p)}\|f\|_{L^{p}(\mathbb{C})} \leq\left\|\boldsymbol{\partial} \bar{\partial}^{-1} f\right\|_{L^{p}(\mathbb{C})} \leq B(p)\|f\|_{L^{p}(\mathbb{C})}, \quad f \in L^{p}(\mathbb{C}),
$$

which we recognize as (1.2). For this reason, the constants cannot be improved.

Acknowledgements. The author thanks Ioannis Parissis, Eero Saksman, and Michael Benedicks for extensive discussions. In addition, he thanks Parissis for several conversations regarding Hardy's inequality and the Cauchy operators of Subsection 1.7. He also thanks the referee for reading the paper carefully, and his thoughtful remarks, which helped to improve the presentation. 


\section{References}

[1] Abramowitz, M., and I. A. Stegun (eds.): Handbook of mathematical functions with formulas, graphs, and mathematical tables. - Dover Publications, reprint of the 1972 edition, New York, 1992.

[2] Astala, K., T. Iwaniec, and G. Martin: Elliptic partial differential equations and quasiconformal mappings in the plane. - Princeton Math. Ser. 48, Princeton Univ. Press, Princeton, NJ, 2009.

[3] Baernstein, A., II, and S. J. Montgomery-Smith: Some conjectures about integral means of $\partial f$ and $\bar{\partial} f$. - In: Complex Analysis and Differential Equations, Proc. of Wallenburg Symposium in Honor of Matts Essén (Uppsala, 1997), edited by C. Kiselman, Acta Univ. Upsaliensis Skr. Uppsala Univ. C Organ. Hist. 64, Uppsala Univ., Uppsala, 1999, 92-109.

[4] Bañuelos, R., and P. JanakiRaman: $L^{p}$-bounds for the Beurling-Ahlfors transform. - Trans. Amer. Math. Soc. 360, 2008, 3603-3612.

[5] Baranov, A., and H. Hedenmalm: Boundary properties of Green functions in the plane. Duke Math. J. 145:1, 2008, 1-24.

[6] Davies, E. B.: Spectral theory and differential operators. - Cambridge Stud. Adv. Math. 42, Cambridge Univ. Press, Cambridge, 1995.

[7] Dragičević, O., and A. Volberg: Bellman function, Littlewood-Paley estimates and asymptotics for the Ahlfors-Beurling operator in $L^{p}(\mathbb{C})$. - Indiana Univ. Math. J. 54, 2005, 971-995.

[8] Hedenmalm, H., I. Parissis, and E. Saksman: Private communication.

[9] IwAnieC, T.: Extremal inequalities in Sobolev spaces and quasiconformal mappings. - Z. Anal. Anwendungen 1, 1982, 1-16.

[10] Magnus, W., F. Oberhettinger, and R. P. Soni: Formulas and theorems for the special functions of mathematical physics. Third enlarged edition. - Grundlehren Math. Wiss. 52, Springer-Verlag, New York, 1966.

[11] MaZ'JA, V. G.: Sobolev spaces. - Springer Ser. Soviet Math., translated from the Russian by T. O. Shaposhnikova, Springer-Verlag, Berlin, 1985.

[12] Stein, E. M.: Harmonic analysis: real-variable methods, orthogonality, and oscillatory integrals. - Princeton Math. Ser. 43, Monographs in Harmonic Analysis III, Princeton Univ. Press, Princeton, NJ, 1993.

[13] Suzuki, N.: Nonintegrability of superharmonic functions. - Proc. Amer. Math. Soc. 113:1, $1991,113-115$.

Received 17 February 2010 